\title{
Bacteriemia en pacientes oncológicos. Experiencia en un hospital pediátrico
}

\author{
Daniela Cortez, Natalie Rodríguez, Dona Benadof, Agustín Zamorano y Juan Tordecilla
}

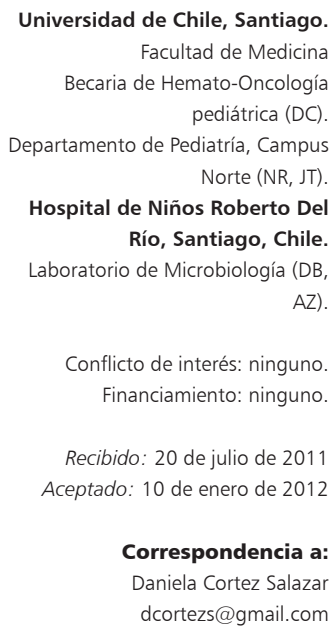

Conflicto de interés: ninguno. Financiamiento: ninguno.

Recibido: 20 de julio de 2011 Aceptado: 10 de enero de 2012

Correspondencia a: Daniela Cortez Salazar dcortezs@gmail.com

\section{Bacteremia in cancer patients. Experience in a pediatric hospital}

The use of intensive chemotherapy has improved survival of children with cancer. However, this is associated to severe and maintained neutropenia, increasing risks of severe infections like bacteremia. Aim: To update information on microorganisms involved in bloodstream infections in cancer patients and their antimicrobial resistance patterns during the last 3 years in our hospital, comparing it with our previous experience and with other Chilean centres. Material and Methods: Analysis of positive blood cultures belonging to cancer patients during 2006-2008 registered in the Microbiology Lab at the Roberto Del Rio Children’s Hospital. Results: In 52 patients, 96 blood cultures yielded bacteria: 59.4\% gram positive cocci and 34.4\%, gram negative rods. Coagulase negative Staphylococci (CNS) were the most frequent bacteria isolated and enterobacteria were in the second place. Susceptibility to cloxacillin was $11 \%$ in CNS and $70 \%$ in Staphylococcus aureus isolates. Enterobacteria maintained susceptibility to third generation cephalosporins and aminoglycosides. Conclusion: Despite the low sensitivity of CNS to cloxacillin, the empirical antibiotic treatment in our unit must include cloxacillin because of the high susceptibility of S. aureus. Switching to vancomycin should be considered only if SCN is isolated or there is an unfavorable evolution.

Key words: Bacteriemia, Pediatrics, etiology, infections, cancer.

Palabras clave: Bbacteriemia, Pediatría, etiología, infección, cáncer.

\section{Introducción}

$\mathrm{E}$ 1 uso de protocolos de quimioterapia más intensivos ha mejorado en forma notable la sobrevida de los niños con cáncer en nuestro país. Sin embargo, esto favorece la aparición de neutropenias intensas y mantenidas, lo que aumenta el riesgo de infecciones graves. Las infecciones asociadas a neutropenia constituyen una de las principales complicaciones de los pacientes oncológicos pediátricos, representando la causa más frecuente de morbilidad y mortalidad en este grupo de niños. En nuestro país, el Comité de Infectología del Programa Infantil Nacional de Drogas Antineoplásicas (PINDA) ha formulado recomendaciones de diagnóstico y tratamiento ajustadas a nuestra realidad nacional ${ }^{1-3}$.

El inicio precoz de un tratamiento antimicrobiano empírico y de amplio espectro ha demostrado ser una medida fundamental para disminuir la mortalidad en este grupo de pacientes. Sin embargo, esta modalidad de tratamiento puede asociarse a la aparición de resistencia a antimicrobianos, sobre-infecciones fúngicas y toxicidad por antimicrobianos ${ }^{4}$.

El conocimiento del tipo y frecuencia de los microorganismos responsables de bacteriemia y su perfil de susceptibilidad antimicrobiana en cada centro hospitalario es importante para establecer el tratamiento empírico más adecuado. Nuestro grupo publicó, en 1994 y 1998, estudios en que los microorganismos aislados con mayor frecuencia en los hemocultivos de pacientes con neutropenia febril fueron cocáceas grampositivas (Staphylococcus coagulasa negativa -SCN- y Staphylococcus aureus) disminuyendo la frecuencia de bacilos gramnegativos, que fueran considerados los agentes etiológicos más importantes de bacteriemias en estos pacientes en la década de los 80. De acuerdo a esto, se planteó el uso de esquemas que incluyeran un antimicrobiano antiestafilocóccico (cloxacilina) más una cefalosporina de $3^{\mathrm{a}}$ generación que cubra bacilos gramnegativos (cefotaxima) y un aminoglucósido (amikacina) para ampliar el espectro sobre especies no fermentadoras. Un estudio multicéntrico nacional, realizado en cinco centros oncológicos pediátricos de Santiago, publicado el año 2001, demostró la aparición de resistencia a meticilina en 55\% de las cepas de SCN y en $44 \%$ de las cepas de $S$. aureus, aisladas en hemocultivos ${ }^{5-8}$.

Desde esa fecha, nuestro centro ha utilizado el mismo esquema antimicrobiano empírico al ingreso de los pacientes con neutropenia febril o sospecha de infección sistémica, aunque no presenten neutropenia. Hemos modificado dicha terapia en caso de evolución desfavorable y/o de acuerdo al resultado de los cultivos, utilizando antimicrobianos de segunda línea (vancomici- 
na, ceftazidima, imipenem) y antifúngicos (anfotericina y/o voriconazol), según las recomendaciones del Comité de Infectología del PINDA ${ }^{9}$.

Considerando estos antecedentes, nuestro objetivo fue actualizar la información sobre los microorganismos implicados en las infecciones del torrente circulatorio en los pacientes oncológicos atendidos en nuestro hospital, comparar con los resultados de nuestra institución y nacionales antes publicados y describir su patrón de resistencia antimicrobiana, con la finalidad de evaluar una eventual modificación a las recomendaciones de tratamiento empírico inicial vigentes.

\section{Material y Método}

Se revisaron los registros de hemocultivos del Laboratorio de Microbiología del Hospital de Niños Roberto Del Río en el periodo comprendido entre el 1 de enero del 2006 y el 31 de diciembre del 2008, seleccionando aquellos con resultado positivo que se obtuvieron de pacientes con diagnóstico de enfermedad oncológica.

\section{Definiciones}

Se consideró hemocultivo positivo a:

- El aislamiento único de cualquier especie usualmente reconocida como patógena en estos pacientes

- Al menos dos hemocultivos positivos para microorganismos propios de la microbiota cutánea como: SCN, Micrococcus sp, Corynebacterium sp, Propionibacterium sp y Bacillus sp. Se registró como duplicación el aislamiento del mismo agente, con la misma sensibilidad, en el mismo paciente en forma consecutiva (menos de 7 días entre ambos aislamientos). En estos casos se consignó sólo una vez el microorganismo aislado, asumiendo que cada cepa aislada de un hemocultivo estaba en correspondencia con un episodio febril o infeccioso por paciente.

Se consideraron como contaminados y se excluyeron del análisis, aquellos hemocultivos en que se aisló una bacteria propia de la piel en uno de dos hemocultivos ${ }^{8}$. Así mismo se excluyeron del análisis los casos donde sólo existía hemocultivo positivo tomado desde catéter venoso central (CVC), sin acompañarse de hemocultivos periféricos positivos, por no cumplir con los criterios de bacteriemia relacionada a $\mathrm{CVC}^{10-12}$.

En todos los casos con hemocultivos positivos se revisaron las fichas clínicas de los pacientes y se registró: edad, sexo, patología de base, asociación con neutropenia intensa (definida como recuento absoluto de neutrófilos [RAN] $<500 / \mathrm{mm}^{3}$ ), presencia de CVC y uso de profilaxis antimicrobiana.

Durante todo el periodo evaluado se utilizaron hemocultivos automatizados BacT/ALERT® (bioMérieux).
Los registros del laboratorio incluyeron género y especie bacteriana, además del estudio de susceptibilidad a antimicrobianos. Los estudios de susceptibilidad in vitro fueron realizados por método de Kirby Bauer ${ }^{13}$ durante los años 2006 y 2007, en el año 2008 por método automatizado con VITEK 1® (bioMérieux); las interpretaciones de la susceptibilidad están basadas en las tablas del CLSI del año correspondiente. La detección de $\beta$-lactamasa de espectro extendido fue confirmada por método de difusión según CLSI ${ }^{14}$.

\section{Análisis estadístico}

Los datos fueron recolectados en planilla excell y analizados en programa SPSS 19.

\section{Resultados}

En el período estudiado se obtuvieron 167 hemocultivos positivos de pacientes oncológicos hospitalizados por cuadros febriles, asociados o no a neutropenia. Se dejaron fuera de análisis 72 hemocultivos por no cumplir con los criterios de inclusión: 37 fueron aislados obtenido sólo a través de un CVC, 21 se consideraron como contaminación y 14 casos fueron considerados una duplicación.

El análisis final se realizó en un total de 95 hemocultivos, con 96 aislados bacterianos, que correspondían a 52 pacientes.

Sólo en un caso se evidenció aislamiento de dos agentes como se detalla más adelante. Del total de las cepas aisladas, 34,4\% correspondieron al año 2006, 36,5\% al año 2007 y 29,2\% al año 2008.

Características demográficas y clínicas (Tabla 1). El diagnóstico más frecuente fue leucemia linfoblástica aguda, el promedio de edad al momento del episodio de bacteriemia fue de 7 años y 2 meses (rango: 1 mes - 17

\begin{tabular}{|c|c|c|}
\hline Género & $\mathbf{n}$ & $\%$ \\
\hline Femenino & 23 & 44,2 \\
\hline Total & 52 & 100 \\
\hline \multicolumn{3}{|l|}{ Diagnóstico } \\
\hline Leucemias agudas (linfoblásticas, mieloblásticas) & 22 & 42,3 \\
\hline Recaída de leucemia & 10 & 19,2 \\
\hline Neuroblastoma & 3 & 5,8 \\
\hline Histiocitosis de células de Langerhans & 3 & 5,8 \\
\hline Linfomas & 3 & 5,8 \\
\hline PNET & 3 & 5,8 \\
\hline \multirow[t]{2}{*}{ Otras neoplasias } & 8 & 15,4 \\
\hline & 52 & 100 \\
\hline
\end{tabular}




\begin{tabular}{|lcc|}
\multicolumn{2}{|c|}{$\begin{array}{c}\text { Tabla 2. Microorganismos aislados en } 96 \text { bacteriemias } \\
\text { en pacientes oncológicos pediátricos. } \\
\text { Hospital Roberto Del Río. 2006-2008 }\end{array}$} \\
Cocaceas grampositivas & n & $\%$ \\
Staphylococcus coagulasa negativa & 29 & 30,2 \\
Streptococcus grupo viridans & 13 & 13,5 \\
Staphylococcus aureus & 10 & 10,4 \\
Streptococcus pneumoniae & 4 & 4,2 \\
Rothia mucilaginosa & 1 & 1,0 \\
& 57 & 59,4 \\
Bacilos gramnegativos & & \\
Escherichia coli & 11 & 11,5 \\
Klebsiella spp & 9 & 9,4 \\
Enterobacter spp & 8 & 8,3 \\
Pseudomonas aeruginosa & 4 & 4,2 \\
Stenotrophomonas maltophilia & 1 & 1,0 \\
& 33 & 34,4 \\
Otros & & \\
Bacillus sp. & 1 & 1,0 \\
Psychrobacter inmovilis & 1 & 1,0 \\
& 2 & 2,0 \\
Hongos & & \\
Candida spp & 3 & 3,1 \\
Aspergillus spp & 1 & 1,0 \\
& 4 & 4,2 \\
Total & 960 \\
\hline
\end{tabular}

Tabla 3. Microorganismos aislados en bacteriemias en pacientes oncológicos pediátricos tratados en Hospital Roberto Del Río. Comparación de tres períodos. 1988-2008

\begin{tabular}{|c|c|c|c|c|c|c|}
\hline & \multicolumn{2}{|c|}{$\begin{array}{l}\text { Tordecilla J. y } \\
\text { cols. }^{5}\end{array}$} & \multicolumn{2}{|c|}{$\begin{array}{l}\text { Tordecilla J. y } \\
\text { cols. }{ }^{7}\end{array}$} & \multicolumn{2}{|c|}{ Reporte actual } \\
\hline Años del estudio & \multicolumn{2}{|c|}{$1988-1992$} & \multicolumn{2}{|c|}{$1993-1996$} & \multicolumn{2}{|c|}{$2006-2008$} \\
\hline Período de revisión & \multicolumn{2}{|c|}{46 meses } & \multicolumn{2}{|c|}{33 meses } & \multicolumn{2}{|c|}{36 meses } \\
\hline Pacientes (n) & \multicolumn{2}{|c|}{84} & \multicolumn{2}{|c|}{113} & \multicolumn{2}{|c|}{52} \\
\hline Episodios de neutropenia & \multicolumn{2}{|c|}{149} & \multicolumn{2}{|c|}{179} & \multicolumn{2}{|c|}{58} \\
\hline Bacteriemias $\left({ }^{*}\right)$ & \multicolumn{2}{|c|}{$18(12 \%)$} & \multicolumn{2}{|c|}{$30(16,8 \%)$} & \multicolumn{2}{|c|}{96} \\
\hline Etiologías de bacteriemias & $\mathrm{n}$ & $\%$ & $\mathrm{n}$ & $\%$ & $\mathrm{n}$ & $\%$ \\
\hline S. coagulasa negativa & 2 & 11,1 & 12 & 40 & 29 & 30,2 \\
\hline Staphylococcus aureus & 7 & 38,9 & 5 & 16,7 & 10 & 10,4 \\
\hline Streptococcus spp & 1 & 5,6 & 4 & 13,3 & 17 & 17,7 \\
\hline Escherichia coli & 1 & 5,6 & 3 & 10 & 11 & 11,5 \\
\hline Klebsiella spp & 2 & 11,1 & 2 & 6,7 & 9 & 9,4 \\
\hline Pseudomonas aeruginosa & 2 & 11,1 & 1 & 3,3 & 4 & 4,2 \\
\hline Candida spp & 3 & 16,6 & 3 & 10 & 3 & 3,1 \\
\hline Otros & 0 & 0 & 0 & 0 & 13 & 13,5 \\
\hline
\end{tabular}

$\left.{ }^{*}\right)$ Los dos primeros reportes corresponden a episodios de neutropenia en los cuales la bacteriemia se presentó en porcentajes expresados entre paréntesis. En el reporte actual se tomaron todas las bacteriemias que se presentaron en pacientes oncológicos pediátricos en el periodo de tiempo descrito. años 5 meses). Sesenta por ciento de las bacteriemias se asociaron a neutropenia y de éstas casi $47 \%$ fueron catalogadas como neutropenias febriles de alto riesgo, de acuerdo al Consenso del Comité de Infectología del PINDA. El 92\% de los eventos se relacionaba con la presencia de CVC, ya fuese transitorio o con reservorio.

Se consignó uso de profilaxis antimicrobiana en los 7 días previos a la bacteriemia en 91 de los 96 aislados. De éstos, 88\% tenía como antecedente el uso de cotrimoxazol y 15,4 \% el uso de amoxicilina por tratarse de pacientes con protocolos en los que se utilizaron altas dosis de citosina arabinósido.

\section{Hallazgos microbiológicos}

Se aislaron cocáceas grampositivas en $59,4 \%$ de los hemocultivos, 34,4\% fueron bacilos gramnegativos, 4,2\% hongos y $1 \%$ correspondió a bacilos gram positivos (Tabla 2). En el grupo de cocáceas grampositivas, se recuperaron, en orden de frecuencia, SCN, Streptococcus grupo viridans, SA y Streptococcus pneumoniae (Tabla 2). En el caso de los bacilos gramnegativos predominaron las enterobacterias como Escherichia coli, Klebsiella sp y Enterobacter sp, en ese orden de frecuencia. Los bacilos gramnegativos no fermentadores se aislaron en 5,2\% del total de bacteriemias, predominando en este grupo Pseudomonas aeruginosa (Tabla 2). Sólo en un episodio se aislaron dos microorganismo y correspondían a Enterobacter aerogenes y Klebsiella oxytoca. Se observaron 4 fungemias, 3 causadas por Candida sp; una de ellas fue identificada como Candida albicans. En un episodio se aisló Aspergillus sp.

\section{Susceptibilidad in vitro}

En el grupo de cocáceas grampositivas (57 aislados), SCN (n: 29) y S. aureus (n: 10) tuvieron una susceptibilidad a cloxacilina de 11 y 70\%, respectivamente. En el grupo de enterobacterias (28 aislados), 64\% fueron susceptibles a cefalosporinas de tercera generación; las cepas resistentes correspondían, en su mayoría, a microorganismos productores de $\beta$-lactamasas de espectro extendido (BLEE). En el caso de los aminoglucósidos, amikacina y gentamicina presentaron actividad in vitro sobre 71 y 79\% de las cepas, respectivamente. Ciprofloxacina fue activa sobre $85 \%$ de ellas.

\section{Discusión}

El uso de esquemas de quimioterapia más agresivos en el tratamiento de los niños con cáncer aumenta el riesgo de neutropenia e infección. Es así como en los episodios de neutropenia febril, la bacteriemia se observa en $20 \%$ de los casos y en los pacientes no neutropénicos sólo en 1 a 6\%, cifras descritas tanto en la experiencia nacional 
como internacional ${ }^{9,15}$. A esto se suma que en la actualidad, prácticamente todos los pacientes con patología oncológica son portadores de un acceso venoso central, otro factor de riesgo para desarrollar una bacteriemia. Frente al riesgo de infección bacteriana invasora, la conducta de iniciar precozmente un tratamiento antimicrobiano de amplio espectro en forma empírica, de acuerdo a las recomendaciones del Comité de Infectología del PINDA, ha permitido mejorar la sobrevida de este grupo de pacientes ${ }^{1,16,17}$.

La mayoría de las publicaciones existentes describen los microorganismos aislados en pacientes con neutropenia febril. En este estudio se muestran las bacteriemias ocurridas en un período de tres años en el Hospital Roberto Del Río en pacientes oncológicos, con o sin neutropenia (estos últimos representan menos de la mitad de los casos estudiados), la mayoría portadores de CVC, en el contexto evolutivo de un cuadro febril.

La distribución de los agentes muestra que la mayor frecuencia está dada por SCN, Streptococcus grupo viridans, S. aureus, E. coli, Klebsiella sp y Enterobacter sp, dando cuenta de casi $84 \%$ de los aislados. Este hallazgo confirma la tendencia observada en la literatura médica extranjera, nacional y también en nuestra institución en las últimas dos décadas ${ }^{18,19}$.

SCN es el agente más frecuente en nuestra experiencia $(30,2 \%)$ y el aumento de su frecuencia está relacionado con el uso de reservorios centrales. En el estudio previo de nuestro grupo (Tabla 3), del período 1988-1992, hubo 18 agentes aislados de 298 hemocultivos y se encontró SCN sólo en dos pacientes, hallazgo atribuible a que en ese período no se utilizaban de rutina accesos venosos centrales permanentes. De hecho, solamente 5 de 84 pacientes los portaban. Además se aislaron en 5 casos bacilos gramnegativos que correspondían a: Klebsiella sp, Pseudomonas sp y Escherichia coli ${ }^{5}$.

En una ulterior experiencia del Hospital Roberto Del Río (1993 -1996) con 113 pacientes que presentaron 179 episodios de neutropenia febril, hubo bacteriemia en 30 casos y en esta serie, el agente más frecuente sí fue SCN (40\%) seguido por S. aureus (16,7\%), E. coli (7,5\%), Klebsiella spp (6,6\%) y C. albicans $(6,6 \%)$. Ya en ese estudio, el aislamiento de Streptococcus grupo viridans alcanzó a 6\%. En dicha publicación, 29 pacientes eran portadores de CVC y 4 de ellos presentaron infección del tracto sanguíneo asociada a CVC: 3 por SCN y 1 por Streptococcus grupo $\mathrm{D}^{7}$.

Payá y cols., describieron los agentes causantes de infecciones del torrente circulatorio en niños con cáncer en cinco hospitales de Santiago entre los años 1994 y 1998. Del total de agentes aislados, 224 cepas correspondían a hemocultivos de pacientes del Hospital Roberto Del Río y también entonces SCN fue el agente más aislado, seguido de $S$. aureus. Este segundo mostró una resistencia a meticilina de $61 \%$, mientras que en SCN no se efectuó estudio de susceptibilidad por no poder diferenciar las cepas contaminantes. El 15\% de las enterobacterias tenía resistencia a gentamicina y amikacina, y entre 20 y $26 \%$ a cefalosporinas de tercera generación. Este estudio concluyó que los agentes involucrados en las bacteriemias se mantienen sin variación en el mediano plazo, en su mayoría microorganismos grampositivos, y que los hallazgos más trascendentes se encuentran en los patrones de susceptibilidad bacteriana, con aumento de resistencia a meticilina en $S$. aureus y SCN; lo mismo se observa en la resistencia de enterobacterias a cefalosporinas de tercera generación y de Streptococcus sp. a penicilina. En base a estos hallazgos, los autores sugirieron que en los casos de neutropenia febril catalogados de alto riesgo, con criterios para plantear una enfermedad invasora, se iniciara un esquema antimicrobiano que incluyera un agente efectivo contra Staphylococcus sp. resistente a meticilina, asociado a un aminoglucósido y a una cefalosporina de tercera generación ${ }^{8}$.

En el presente estudio, que comprende 57 aislados de cocáceas grampositivas, SCN mostró una sensibilidad de sólo $11 \%$ a cloxacilina, mientras que $S$. aureus fue susceptible a este antimicrobiano en $70 \%$ de los casos. En los 28 aislamientos de enterobacterias, la sensibilidad a cefalosporinas de tercera generación alcanzó a 64\%, la que aumentaba hasta $79 \%$ para aminoglucósidos, observándose un leve aumento de la resistencia a aminoglucósidos y cefalosporinas de tercera generación. El patrón de susceptibilidad observado representa en forma significativa el grupo analizado, ya que se recomienda que en los estudios de susceptibilidad bacteriana, los grupos por género bacteriano analizado deben incluir al menos 30 cepas $^{18}$.

En otra experiencia nacional, sobre 100 episodios de neutropenia febril, se observó que de los microorganismos aislados en hemocultivos, SCN en su mayoría eran resistentes a meticilina, mientras que $S$. aureus mostró ausencia de resistencia y $E$. coli resultó multisensible. El tratamiento antimicrobiano inicial usado es similar al nuestro y hubo cambio o ampliación del esquema utilizado en forma empírica en 52\% de los casos ${ }^{21}$.

En nuestros pacientes iniciamos tratamiento con cloxacilina, amikacina y cefotaxima, lo que cubriría en forma razonable a los agentes aislados, considerando que S. aureus sigue siendo un microorganismo frecuente en nuestro medio. No se utilizan, de comienzo, antifúngicos debido a que las infecciones fúngicas no se presentan habitualmente al inicio del cuadro febril. Considerando todos estos antecedentes, creemos que en los cuadros febriles en niños con patología oncológica, especialmente neutropénicos y con un alto riesgo de una infección bacteriana invasora, el esquema antimicrobiano empírico de ingreso está vigente. Esto debido a que tanto en el presente trabajo, 
como en la experiencia del grupo nacional PINDA, SCN y $S$. aureus siguen siendo los principales microorganismos grampositivos recuperados en los hemocultivos, manteniendo $S$. aureus la susceptibilidad a cloxacilina durante estos años. En caso de hemocultivo positivo a SCN o evolución clínica desfavorable, se debería cambiar cloxacilina por vancomicina, pero manteniendo la cefalosporina y el aminoglucósido inicial porque aún estamos ante un paciente en riesgo, por la neutropenia, de co-infección o superinfección bacteriana. Esta conducta además permite evitar el uso excesivo de vancomicina y la aparición de cepas resistentes a ella.

\section{Resumen}

El uso de quimioterapia más intensiva ha mejorado la sobrevida de los niños con cáncer. Sin embargo, esto se asocia a neutropenia intensa y mantenida, aumentando el riesgo de infecciones graves como bacteriemias. Objetivo: Actualizar la información sobre los microorganismos implicados en las infecciones del torrente circulatorio en pacientes oncológicos atendidos en nuestro hospital, comparar con la literatura médica y describir el patrón de resistencia antimicrobiana. Material y Métodos: Se revisaron los registros de hemocultivos del Laboratorio de Microbiología del Hospital de Niños Roberto Del Río entre los años 2006 y 2008, seleccionando aquellos con resultado positivo y que pertenecieran a pacientes con cáncer. Resultados: En 52 pacientes, 96 hemocultivos resultaron positivos: cocáceas grampositivas 59,4\% (Staphylococcus coagulasa negativa-SCN fue el más frecuente); bacilos gramnegativos $34,4 \%$, predominando las enterobacterias, en segundo lugar. Se observó en SCN una susceptibilidad a cloxacilina de $11 \%$ y en Staphylococcus aureus de $70 \%$. Las enterobacterias mantuvieron una susceptibilidad estable para cefalosporinas de tercera generación y aminoglucósidos. Conclusión: A pesar de la baja susceptibilidad de SCN a cloxacilina, el tratamiento antimicrobiano empírico de primera línea en nuestra unidad debe incluir cloxacilina dada la alta susceptibilidad de $S$. aureus, y el cambio a vancomicina debería plantearse frente al aislamiento de SCN o evolución desfavorable.

\section{Referencias}

1.- Santolaya M E, Álvarez A M, Becker A, Cofré J, Enríquez N, O’Ryan M, et al. Prospective, multicenter evaluation of risk factors associated with invasive bacterial infection in children with cancer, neutropenia and fever. J Clin Oncol 2001; 19: 3415-21.

2.- Santolaya ME, Álvarez AM, Avilés L, Becker A, Cofré J, Enríquez N, et al. Prospective evaluation of a model of prediction of invasive bacterial infection risk among children with cancer, fever and neutropenia. Clin Infect Dis 2002; 35: 678-83.

3.- Santolaya M E, Álvarez A M, Avilés C, Becker A, Cofré J, Payá E, et al. Tratamiento selectivo de los episodios de neutropenia febril en niños con cáncer. Comité de Infectología, Programa Infantil Nacional de Drogas Antineoplásicas (PINDA). Rev Chil Infectol 2004; 21 (3): 213-22.

4.- Pizzo P A. Management of fever in patients with cancer and treatment-induced neutropenia. N Engl J Med 1993; 328: 1323-32.

5.- $\quad$ Tordecilla J, Campbell M, Joannon P. Neutropenia febril en niños con cáncer. Rev Chil Pediatr 1994; 65 (3): 149-53.

6.- Tordecilla J, Campbell M, Joannon P, Rodríguez N. Alta precoz de niños con cáncer y neutropenia febril. Rev Chil Pediatr 1994; 65 (5): 260-3.

7.- $\quad$ Tordecilla J, Campbell M, Joannon P, Rizzardini C, Soto V. Criterios de alta precoz en niños con cáncer y neutropenia febril. Rev Chil Pediatr 1998; 69 (6): 247-51.

8.- Payá E, Álvarez AM, Avilés C, Cofré J, Enríquez N, Salgado C, et al. Agentes causantes de infecciones del torrente circulatorio en niños con cáncer en cinco hospitales de Santiago (1994-1998). Rev Med Chile 2001; 129 (11): 1297-304.

9.- Santolaya ME, Rabagliati R, Bidart T, Payá E, Guzmán AM, Morales R, et al . Consenso Manejo racional del paciente con cáncer, neutropenia y fiebre. Rev. Chil. Infectol. 2005; 22 (Supl 2): S79.

10.- Mermel L, Allon M, Bouza E, Craven D, Flynn P, O'Grady N, et al. Clinical Practice: Guidelines for the Diagnosis and Management of Intravascular Catheter-Related Infection: 2009 Update by the Infectious Diseases Society of America. Clin Infect Dis. 2009; 49(1): 1-45.

11.- Acuña M, O’Ryan M, Cofré J, Álvarez I, Benadof D, Rodríguez P et al. Differential time to positivity and quantitative cultures for noninvasive diagnosis of catheter-related blood stream infection in children. Pediat Infect Dis J. 2008; 27 (8): 681-5.

12.- García P, Payá E, Olivares R, Cotera A, Rodríguez J, Sanz M. Diagnóstico de las infecciones asociadas a catéteres vasculares centrales. Rev Chil Infectol 2003; 20 (1): 41-50.

13.- Procedimientos Técnicos de Laboratorio Clínico. Instituto de Salud Pública de Chile. 1994. Volumen I.

14.- CLSI. Performance standards for antimicrobial sensibility testing; informational supplement
CLSI document M100-S21. Wayne, PA: Clinical and Laboratory Standards Institute. January 2006, January 2007, January 2008.

15.- Yadegarynia D, Tarrand J, Raad I, Ralston K. Current spectrum of bacterial infections in patients with cancer. Clin Infect Dis 2003; 37 (8): 1144-5

16.- Rackoff W, Gonin R, Robinson C, Kreissman $S$, Breitfeld P. Predicting the risk of bacteriemia in children with fever and neutropenia. J Clin Oncol 1996; 14: 919-24.

17.- Kelly M, Vivier P, Panken T, Schwartz C. Bacteriemia in febrile non neutropenic pediatric oncology patients. Pediatr Blood Cancer 2009; DOI 10.1002: 1-5.

18.- Castagnola F, Fontana V, Caviglia L, Caruso S, Faraci M, Fioredda F, et al. A prospective study on the epidemiology of febrile episodes during chemotherapy-induced neutropenia in children with cancer or after hemopoietic stem cell transplantation. Clin Infect Dis 2007; 15: 1296-304.

19.- Meckler G, Lindemulder S. Fever and neutropenia in pediatric patients with cancer. Emerg Med Clin North Am 2009; 27: 525-44.

20.- Silva F, Cifuentes M, Pinto ME. Resultados de la vigilancia de susceptibilidad antimicrobiana en Chile: consolidando una red. Rev Chil Infectol 2011; 28 (1):19-27.

21.- Muñoz E, Ossa JC, Villarroel M, Santolaya ME. Tratamiento antimicrobiano en niños con neutropenia febril de alto riesgo. Rev Chil Pediatr 2008; 79(4): 381-7. 